\title{
Chemical modification of chitosan film via surface grafting of citric acid molecular to promote the biomineralization
}

\author{
Yang Liu *a, b, c, Xin Shen ${ }^{\text {a }}$, Huan Zhou a ${ }^{\text {, }}$ Yingjun Wang ${ }^{\mathrm{c}}$, Linhong Deng *a \\ ${ }^{a}$ Institute of Biomedical Engineering and Health Sciences, Changzhou University, Changzhou 213164, China \\ ${ }^{b}$ Engineering Research Center of Nano-Geo Materials of Ministry of Education China University of Geosciences, \\ Wuhan 430074,China \\ ${ }^{c}$ School of Materials Science and Engineering, South China University of Technology, Guangzhou 510641, China
}

Fax:0519-86330103; Tel: +86 18861259625; E-mail: liuyang@cczu.edu.cn; dlh@cczu.edu.cn

* Author for correspondence:

Yang Liu, Ph.D., Institute of Biomedical Engineering and Health Sciences, Changzhou University, Changzhou,

Jiangsu, 213164, P. R. China, Email: liuyang@cczu.edu.cn

Linhong Deng, Ph.D., Institute of Biomedical Engineering and Health Sciences, Changzhou University,

Changzhou, Jiangsu, 213164, P. R. China, Email: dlh@ @czu.edu.cn

(C) 2016. This manuscript version is made available under the Elsevier user license http://www.elsevier.com/open-access/userlicense/1.0/ 


\begin{abstract}
We develop a novel chitosan-citric acid film (abbreviated as CS-CA) suitable for biomedical applications in this study. In this CS-CA film, the citric acid, which is a harmless organic acid has been extensively investigated as a modifying agent on carbohydrate polymers, was cross-linked by 1-Ethyl-3-(3-dimethyl aminopropyl) carbodiimide (EDC) and N-hydroxysuccinimide (NHS) onto the surface of chitosan (CS) film. Fourier transform infrared spectroscopy (FTIR) and X-ray photoelectron spectroscopy (XPS) confirms the graft copolymerization of the modified chitosan film (CS-CA). Surface wettability, moisturizing performance, the capacity of mineralization in vitro and biocompatibility of the films were characterized. After modification, this CS-CA film has good hydrophilicity. It is very evident that the citric acid grafting treatment significantly promotes the biomineralization of the chitosan based substrates. Cell experiments show that the MC3T3-E1 osteoblasts can adhere and proliferate well on the surface of CS-CA film. This CS-CA film, which can be prepared in large quantities and at low cost, should have potential application in bone tissue engineering.
\end{abstract}

Key words: Chitosan; Surface grafting; Citric acid; Chemical modification; Biomineralization. 


\section{Introduction}

Metals are commonly used as biomaterials to replace damaged human bone tissues. However, such implants pose the risk of releasing harmful metal ions, and may induce allergic reactions [1]. To prevent this risk, the surfaces of these implants are usually protected by a bioactive coating [2-6]. Among these coating materials, chitosan possesses outstanding properties, such as non-toxicity, biodegradability, biocompatibility, and antimicrobial [7-11]. As one of the most abundant polysaccharides in crustacean exoskeletons and is commonly found in the cell walls of several fungi and algae, chitosan consists of a large number of hydroxy and amino groups [12]. These features make the chitosan based materials have been used in a wide variety of applications such as nutrition, ophthalmology, orthopaedics, medicinal and cosmetics, etc. [13-16]. However, it's worth noting that in bone tissue engineering, the mineralization process of chitosan based material is slow due to the hydrophobic property of the chitosan film or coating.

Surface graft modification is an effective approach for the functionalization of the target materials, the reactive sites (hydroxyl and amino groups) of the chitosan materials can be used to covalently bond polymer chains, chemical monomers or small molecules to make the biomaterials have special properties. Modification of chitosan material by grafting with poly (vinyl alcohol) [17-19], poly (ethylene glycol) [20], and other compounds has been report. However, these graft molecules have poor biocompatibility and degradability, which affects the long-term biological safety of chitosan-based materials. For that reason, modifications of the chitosan film using biodegradable natural molecules to obtain the excellent physicochemical and biocompatibility of the new substrate are necessary.

One of the organic acids used for surface modification of chitosan film in this research is citric 
acid, which is a common natural small molecule, has been widely used in food, beverage, chemical and medical applications [21-24]. Compared with other substances, molecular composition and the spacer arm of the citric acid molecule makes it has various functions such as balancing the hydrophilicity of biomaterials, participating in hydrogen bonding interaction and improving the ability of deposition of inorganic ions due to increasing number of carboxyl groups [25]. There are some composite materials that containing chitosan and citric acid has been reported, the citric acid was used as crosslinking agent [26], nucleation catalyst [27], and the treatment agent for removing heavy metal ions [28], respectively. The citric acid molecules play an important role in these systems. In this research, we hope to improve the mineralization ability of chitosan-based materials by introducing the carboxyl groups from the citric acid molecule. To improve the hydrophilicity and mineralization ability of the chitosan film, the citric acid was grafted onto the surface of chitosan film by making use of 1-Ethyl-3-(3-dimethyl aminopropyl) carbodiimide (EDC) and N-hydroxysuccinimide (NHS) as the cross-linker. The aim of this study is to investigate how citric acid surface modification influences the surface morphology, moisturizing performance, biomineralization capacity and biocompatibility of the chitosan film.

\section{Material and methods}

\subsection{Materials}

Chitosan (CS) ( $\geq 95 \%$ deacetylated) was purchased from Aladdin Industrial Inc, (Shanghai, China). Citric acid (CA) was obtained from Aladdin Industrial Inc, (Shanghai, China). Acetic acid was supplied by Shenbo chemical co., LTD, (Shanghai, China). Glutaric dialdehyde (GDI) (solution 25\% wt) was provided by Aladdin Industrial Inc, (Shanghai, China). 1-Ethyl-3-(3-dimethyl amino-propyl) carbodiimide (EDC) was purchased from Sass chemical 
technology co., LTD, (Shanghai, China). N-hydroxysuccinimide (NHS) was obtained from Aladdin Industrial Inc, (Shanghai, China). Simulated body fluid (SBF) was prepared following Kokubo and Takamada method [29]. Deionized water was extracted from a water purification system (Millipore S.A.S., USA).

\subsection{Preparation of films}

Firstly, the purified chitosan powder was dissolved in $0.01 \mathrm{~mol} \mathrm{~L}^{-1}$ acetic acid solution and the citric acid was dissolved in deionized water with the concentration of $10 \mathrm{mg} \mathrm{mL}^{-1}$. Secondly, chitosan solution (the mass fraction is about 3\%) was dispensed into petri dish and dried at room temperature to form a dried chitosan film. Thirdly, the air-dried CS film was immersed into a certain amount of citric acid solution. Afterwards, EDC and NHS were added to crosslink the chitosan and citric acid (the mass ratio of EDC:NHS:CS-CA is 1:1:6). The cross-linking process was carried out by stirring the mixture for about 4 hours. After soaking, the CS-CA film was air-dried, rinsed 3 times with Milli-Q water, and air-dried again under constant temperature. For comparison, cross-linked chitosan film without citric acid was prepared as the control group (CS).

Scheme 1 displays the chemical reaction mechanism between the citric acid and chitosan.

\subsection{Fourier transform infrared (FTIR) spectroscope}

The FTIR spectrum of the CS and CS-CA was analysed using Fourier transform infrared-attenuated total reflectance (Nicolet Avatar 370, USA). Before acquiring the transmission infrared spectra of the samples, a background spectrum was collected. All the spectrums were gained between 4000 and $650 \mathrm{~cm}^{-1}$.

\section{$2.4 X$-ray photoelectron spectroscope (XPS)}

XPS measurement was carried out with X-ray spectrometer system, using a monochromatic $\mathrm{Al}$ 
$\mathrm{K} \alpha \mathrm{X}$-ray source $(\mathrm{h} v=1486.6 \mathrm{eV}, 150 \mathrm{~W})$ and $\mathrm{C} 1 \mathrm{~s}$ of $\mathrm{C}-\mathrm{C}(284.6 \mathrm{eV})$ was chose as the reference line. All spectra were obtained using a $202 \mu \mathrm{m}$ diameter analysis area. The wide scanning was gathered with pass energy of $140.00 \mathrm{eV}$ and a step size of $0.25 \mathrm{eV}$, while high-resolution region scans were operated with pass energy of $55 \mathrm{eV}$ at a scan rate of $0.1 \mathrm{eV}$ per step over a range of $1000 \mathrm{eV}$. A Gaussian-Lorentzian function was applied to fit the spectra for each peak, in order to assume the kinds and quantifications of the main elements. Chemical analysis and quantification spectra from the individual peaks of the CS and CS-CA film were obtained.

\subsection{Surface contact angle measurements}

Contact angles (CA) were tested by the sessile drop method. Measurement was carried out on Powereach JC2000D1 with an injection volume of $1 \mu \mathrm{L}$ distilled water as medium. CA was calculated by a circle segment function intersecting with a straight baseline representing the surface $(n=5)$.

\subsection{Swelling test}

Swelling studies were performed for CS and CS-CA using PBS ( $\mathrm{PH}=7.4)$. Water absorption of the films was tested by swelling them in PBS at the normal physiological temperature (about $37^{\circ} \mathrm{C}$ ). Wet weight of the samples was, after gently blotting the membrane surface with filler paper to remove the absorbed water, weighed immediately at different time intervals. The water absorption of the films was calculated by the following formula:

$$
\text { Water absorption }=\left(\mathrm{M}_{\mathrm{t}}-\mathrm{M}_{0}\right) / \mathrm{M}_{\mathrm{t}} \times 100 \% \text {, }
$$

It's worth noting that chitosan have swelling feature after absorbing water, so it's meaningful to measure the dimensional changes of the swelled films. The thickness and surface area of the samples were measured by a micrometer caliper and rules, respectively. Variations of thickness 
and surface area are calculated according to the following formulas:

Thickness increase $=\mathrm{H}_{\mathrm{t}} / \mathrm{H}_{0}$;

Surface area increase $=S_{t} / S_{0}$.

Here, $M_{t}$ is the wet weight of the samples and $M_{0}$ represents the primal dry weight of the sample. $\mathrm{H}_{t}$ and $\mathrm{S}_{\mathrm{t}}$ are the thickness and surface area of the wet samples at target times, respectively. $\mathrm{H}_{0}$ and $\mathrm{S}_{0}$ are the initial thickness and surface area of the dry samples, respectively. The values are expressed as the means \pm standard error $(n=10)$.

\subsection{In vitro biomineralization}

The biomineralization capability of CS and CS-CA was evaluated by in vitro soaking experiments. Both of the two specimens were immersed in simulated body fluid (SBF) and incubated at $37{ }^{\circ} \mathrm{C}$. The incubation solution in culture dishes was replaced with fresh SBF every day. After rinsing by deionized water and air dried, the mineralized CS and CS-CA samples were withdrawn for observation and characterization. The morphology of the specimens was characterized using a FE-SEM (SU8020, HITACHI). Before the observation, the specimens were gold coated and observed at accelerating voltage of $15 \mathrm{kV}$. FTIR-ATR (Nicolet Avatar 370, USA) spectra $\left(4000-400 \mathrm{~cm}^{-1}\right)$ were used to analyze the chemical functional groups of the films. XRD were performed using D/MAX-2400 (Rigaku) diffractometer with $\mathrm{Cu} \mathrm{K \alpha}$ radiation at room temperature from $10^{\circ}$ to $60^{\circ}$ (wide angle).

\subsection{Cell experiments}

\subsubsection{In vitro MC3T3-E1 osteoblasts culture}

The cytocompatibility of the samples were evaluated using MC3T3-E1 osteoblasts, which were supplied by Northwestern polytechnical university. The MC3T3-E1 osteoblasts were cultured in 
Minimum essential medium (GIBCO), supplemented with $10 \%$ fetal bovine serum (Thermo scientific), $100 \mathrm{U} / \mathrm{mL}$ penicillin, $100 \mu \mathrm{g} / \mathrm{mL}$ streptomycin and $0.2 \%$ levofloxacin. The cells were incubated in a humidified atmosphere (MCO-18AIC(UV), Japan) containing 5\% carbon dioxide at $37{ }^{\circ} \mathrm{C}$.

\subsubsection{The response of MC3T3-E1 osteoblasts to the CS-CA film}

Before cell experiments, the CS-CA film was washed three times with PBS under aseptic conditions, then sterilized by ultraviolet radiation for $1 \mathrm{~h}$ and washed by PBS again at last. After disinfection, the wet film was attached in a 48-well tissue culture plate (Corning, UK). The seeded cell density was about 2000 cells $\mathrm{cm}^{-2}$. Then, the cell-seeded film was incubated in a humidified atmosphere $\left(5 \% \mathrm{CO}_{2}, 37^{\circ} \mathrm{C}\right)$. The culture medium was replaced every day. The response of MC3T3-E1 osteoblasts to the samples were examined with an inverted fluorescence microscope (Primo Vert, Zeiss, German). Before observation, the culture medium was replaced.

\subsubsection{The proliferation of MC3T3-Elosteoblasts to the CS and CS-CA film}

The quantitative assay was carried out in 96-well tissue culture plates (Corning, UK). After the samples were attached in 96-well tissue culture plates (Corning, UK). MC3T3-E1 suspension was seeded onto the CS films and CS-CA films ( $\mathrm{n}=10)$, respectively. The seeded cell density was about 2000 cells $\mathrm{cm}^{-2}$. The proliferation activity of the MC3T3-E1 osteoblasts cells to the samples were quantitatively determined by 3-4,5-dimethyl-2-thiazolyl)-2,5-diphenyl-2-H-tetrazolium bromide (MTT) assay.

\subsection{Histological observation}

After the MC3T3-E1osteoblasts were cultured on the CS-CA film for a week, the entire sheets of MC3T3-E1osteoblasts on the CS-CA film were mounted and fixed with $4 \%$ paraformaldehyde 
for 24 hours. The samples were processed and dehydrated step-wise using ethanol, immersed in xylene, and embedded in paraffin. After that, the Paraffin sections were stained with hematoxylin and eosin (H\&E staining, ) using standard histochemical techniques, and viewed under a light microscope (DM5000 B, Leica, Germany).

\subsection{Statistical analysis}

All data are shown as mean \pm standard deviation. Experiments were analyzed using analysis of variance (ANOVA) to determine the significant differences among the groups. Statistical significance was defined as $\mathrm{P}<0.05$.

\section{Results}

\subsection{ATR-FTIR spectra analysis}

Infrared spectroscopy is often employed for the identification of functional group and provides information of a new synthetic polymer. Fig.1 shows the ATR-FTIR spectrum of the CS and CS-CA film. The main characteristic bands associated with chitosan molecular are detected as follows: the amide I band is associated with stretching vibrations of $\mathrm{C}=\mathrm{O}$ and appears at $1642 \mathrm{~cm}^{-1}$; amide II, associated with C-N stretching and N-H vibration, has a band at $1577 \mathrm{~cm}^{-1}$; and the band at $1326 \mathrm{~cm}^{-1}$ of amide III is due to N-H and C-N stretching. Differences between the spectral of the CS and CS-CA film are also evident. As shown in Fig. 1, by moving from CS toward the CS-CA, the spectrum shifts upwards, towards higher absorption intensities. These increases in intensity are more pronounced at citric acid's characteristic peaks, e.g. at $1028 \mathrm{~cm}^{-1}(\mathrm{O}-\mathrm{H})$ and $1065 \mathrm{~cm}^{-1}$ (C-O). Furthermore, some of the characteristic absorption bands of citric acid are identified for CS-CA film. The FTIR spectra of CS-CA depict characteristic citric acid absorption bands at $1150 \mathrm{~cm}^{-1}$ and $894 \mathrm{~cm}^{-1}$, which are attributed to the C-O-C stretching. The ATR-FTIR 
result implies that citric acid has been graft onto the surface of chitosan successfully.

\subsection{XPS analysis}

X-ray photoelectron spectroscopy (XPS) was used to provide the surface chemical information of the resulting films. XPS spectrum of CS and CS-CA is shown in Fig. 2 and the elemental composition analysis is showed in Table 1 . When citric acid was incorporated on the surface of chitosan film, O 1s peak increased significantly. After chemical grafting reaction, the oxygen content increased from $27.45 \%$ to $33.03 \%$, while the nitrogen content decreased from $3.21 \%$ to $1.32 \%$. Correspondingly, the content of $\mathrm{O} / \mathrm{C}$ increased from 0.40 to 0.50 , and that of $\mathrm{N} / \mathrm{C}$ decreased from 0.05 to 0.02 . The increase in percentage content of oxygen demonstrates that citric acid is successfully introduced by chemical grafting.

The high-resolution XPS spectra of C 1s were obtained and peak fittings were performed to further investigate the detailed chemical bonding and compositions of CS and CS-CA (Fig. 3). The C1s spectrum of the samples could be resolved into four main peaks of different carbon species (Fig. 3A and B). The peaks around $283.47 \mathrm{eV}, 284.07 \mathrm{eV}, 285.27 \mathrm{eV}, 287.00 \mathrm{eV}$ are corresponded to $-\mathrm{C}-\mathrm{C}-,-\mathrm{C}-\mathrm{N}-,-\mathrm{C}-\mathrm{O}-$ and $-\mathrm{C}=\mathrm{O}-$, respectively. The atomic percentage and assigned chemical groups for each peak within the region envelopes are listed in Table 2. The ratio of the absorptive peaks area corresponding to $-\mathrm{C}-\mathrm{O}-$ and $-\mathrm{C}=\mathrm{O}$ - increased obviously after the introduction of citric acid. The increases in carboxyl and the new generated amide bond content in the CS-CA film compared with the CS film is because of the cross-linking of chitosan with citric acid through the condensation polymerization of amine and carboxyl.

\subsection{Surface wettability of CS and CS-CA}

Contact angle (CA) is an important index used to evaluate the hydrophilic/hydrophobic property 
of a material. Fig. 4 shows the variation of contact angle versus time of the CS and CS-CA film. Water spreading on the CS film occurred within $45 \mathrm{~s}$ and the contact angle decreased from $98.7 \pm$ $0.5^{\circ}$ to $87.5 \pm 0.7^{\circ}$, revealing the hydrophobic surface of the CS. However, the contact angle of CS-CA film decreased from $83.1 \pm 0.6^{\circ}$ to $69.4 \pm 0.7^{\circ}$. The lower contact angle observed on CS-CA indicates that its surface is polar and more hydrophilic compared to the CS film. As we know, citric acid has three carboxyl groups, successful grafting of citric acid molecules may introduce these functional groups at the surface of CS-CA, which results in a higher molecular polarity of the CS-CA film than that of the CS film, so the CS-CA film exhibited a minor contact angle. Therefore, we concluded that the CS-CA's hydrophilicity is better than that of the CS film. In addition, it's worth noting that the surface hydrophilicity can influence the mineralization process of the biomaterials by influencing the effectiveness of bonding between the substrate and surrounding ions [30-31].

\subsection{Water absorption of CS and CS-CA}

Fig.5 displays the water absorption capacity of CS and CS-CA samples. After the specimens had been placed in PBS for 0.5 hour, the water absorption of the two films tended to be constant. Water absorption percentages for CS and CS-CA is about $57.1 \pm 3.2 \%$ and $69.6 \pm 4.4 \%$, respectively (Fig.5A). The moisturizing ability of CS-CA film is higher than that of CS film. This may be due to the introduction of citric acid has brought a large number of hydrophilic carboxyl groups on the surface of CS-CA film.

It's worth noting that the size of a tissue substitute should be easy to control because of the sizes of wounds are different for each patient. Fig.5B and Fig.5C show the variations of thickness and surface area versus time of the CS and CS-CA film. After the films had been immersed in PBS for 
$0.5 \mathrm{~h}$, the dimensions of the specimens tended to be constant $(\mathrm{n}=10)$. Experimental results show that the thickness and surface area of the as-synthesized CS-CA film are controlled and repeatable. According to the target wet material's thickness and surface area, we can obtain the dry material with the corresponding size easily.

\subsection{The biomineralization capacity of CS and CS-CA}

Biomineralization capacity of the CS and CS-CA samples that after the incubation in simulated body fluids (SBF) was studied. Fig.6. displays the photograph of CS and CS-CA film that had been stored in SBF for 0 and 31 days, respectively. Compared with the CS film (Fig. 6B), significantly more mineralization composition was found on the surface of the CS-CA film (Fig. 6D). In addition, the SEM images in Fig. 7 further suggested that there is no obvious apatite mineralization components on the surface of CS film (Fig. 7C and E); in contrast, a large number of apatite clusters were observed on the surface of CS-CA film (Fig. 7D and G). The citric acid modified chitosan film (CS-CA film) induced significant apatite mineralization in SBF, implying that the abundant carboxyl groups in citric acid not only act as molecular anchor which facilitate the chemical conjugation of chitosan and citric acid but also play a role in the chelation of inorganic ions in SBF. The electrostatic force between $\mathrm{Ca}^{2+}$ and $\mathrm{PO}^{3-}$ cause the formation of amorphous apatite which gradually grows into apatite crystal after soaking in SBF [31]. Moreover, the chemical composition and the accurate crystal type of the Ca-P minerals were further researched. FTIR spectra of the CS-CA film after being soaked are displayed in Fig. 7I, two crystalline $\mathrm{P}=\mathrm{O}$ vibration absorption bands at 1081 and $1260 \mathrm{~cm}^{-1}$ are observed in the spectrum of the reacted CS-CA film. Fig. 7J gives the XRD patterns of the CS-CA film after immersion in SBF. After being soaked, the sample presents apparent peaks at $25.8^{\circ}, 31.7^{\circ}$ and $45.4^{\circ}$, which can be 
assigned to (002), (211) and (203), respectively of crystalline apatite. Collecting the above information, it is very evident from our results that the citric acid grafting treatment significantly influence the biomineralization process of the chitosan based substrates, which enhance the growth rate of apatite on the citric acid grafted chitosan film. Furthermore, compared with the other existing modification methods [32-35], our method is more suitable for the chitosan film or coating materials.

\subsection{The morphology of MC3T3-E1 on the CS-CA film}

Biological compatibility of a material is essential for its application in tissue engineering. Fig.8 shows the morphology of MC3T3-E1 osteoblasts on the CS-CA film at different time points. After the MC3T3-E1 was seeded on the CS-CA film for one day, the cell grew well and proliferated rapidly. The surface of the samples was almost completely covered by MC3T3-E1 72 hours later. Additionally, the cells began to exhibit polygonal or slightly spindle shape on the surface of the CS-CA sample within one day of incubation. In the following days, well interconnection between MC3T3-E1 cells was observed and the morphology of cells on CS-CA film is quite similar to the cell grows on the normal tissues, which demonstrates that the CS-CA film has good cell compatibility.

\subsection{The proliferation of MC3T3-E1 and histological sections of the CS-CA film}

The quantitative analysis of cellular viabilities at the as-synthesized CS-CA film was evaluated using the MTT assay. It is a measure of cell metabolism and the amount of formazan produced is related to the number of living cells. The pristine CS film and blank were chose as the control groups $(\mathrm{n}=10)$. As shown in Fig. 9A, both the modified and unmodified samples show increased absorbance value, the absorbance value at CS-CA group is slightly larger than that at the CS film. 
The cell viability of the CS-CA film is comparable to that of the control, suggesting the excellent biocompatibility of the resulting CS-CA film. Furthermore, fig.9B shows the H\&E staining section of CS-CA film, which has been used for MC3T3-E1 osteoblasts culture for 7 days. The photograph shows that 2-3 layers cells had covered the whole surface of the CS-CA samples. H\&E staining section of CS-CA film indicates that the MC3T3-E1 can be well bonded to the surface of CS-CA film. The results of these studies suggest that the as-synthesized CS-CA film has good biocompatibility.

\section{Conclusions}

Surface of chitosan film were successfully modified using citric acid in this study. FTIR and XPS results confirmed that citric acid had successfully covered the chitosan film's surface. After modification, this CS-CA film has good hydrophilicity and excellent biomineralization ability. It is very evident that the citric acid grafting treatment significantly improves the biomineralization capacity of the chitosan-based substrates. After the introduction of citric acid molecules, a large number of carboxyl groups appear on the surface of CS-CA film makes it has various functions such as balancing the hydrophilicity, participating in hydrogen bonding interaction and improving the ability of deposition of inorganic ions. MC3T3-E1 osteoblasts can grow well and proliferate rapidly on surface of the CS-CA film. H\&E staining section results indicate that the MC3T3-E1 cells can be well bonded to the surface of CS-CA film. Our preliminary results suggest that this novel citric acid modified chitosan coating may have potential use in bone tissue engineering.

\section{Acknowledgements}

This work is supported by the National Science Foundation of China (No. 11532003, 51273072), the Project of Natural Science Research of Higher Education Institutions of Jiangsu Province (NO. 
15KJB310001).

\section{References}

[1] Chang S-H, Chian C-H. Plasma surface modification effects on biodegradability and protein adsorption properties of chitosan films. Applied Surface Science. 2013;282:735-40.

[2] Ballarre J, Manjubala I, Schreiner WH, Orellano JC, Fratzl P, Ceré S. Improving the osteointegration and bone-implant interface by incorporation of bioactive particles in sol-gel coatings of stainless steel implants. Acta Biomaterialia. 2010;6:1601-9.

[3] Chen S-h, Zheng L-z, Xie X-h, Wang X-l, Lai Y-x, Chen S-k, et al. Comparative study of poly (lactic-co-glycolic acid)/tricalcium phosphate scaffolds incorporated or coated with osteogenic growth factors for enhancement of bone regeneration. Journal of Orthopaedic Translation. 2014;2:91-104.

[4] Cordero-Arias L, Cabanas-Polo S, Goudouri OM, Misra SK, Gilabert J, Valsami-Jones E, et al. Electrophoretic deposition of $\mathrm{ZnO} /$ alginate and $\mathrm{ZnO}$-bioactive glass/alginate composite coatings for antimicrobial applications. Materials Science and Engineering: C. 2015;55:137-44.

[5] Pishbin F, Mouriño V, Gilchrist JB, McComb DW, Kreppel S, Salih V, et al. Single-step electrochemical deposition of antimicrobial orthopaedic coatings based on a bioactive glass/chitosan/nano-silver composite system. Acta Biomaterialia. 2013;9:7469-79.

[6] Bellucci D, Sola A, Gentile P, Ciardelli G, Cannillo V. Biomimetic coating on bioactive glass-derived scaffolds mimicking bone tissue. Journal of Biomedical Materials Research Part A. 2012; 100A: 3259-66. 
[7] Qin L, Dong H, Mu Z, Zhang Y, Dong G. Preparation and bioactive properties of chitosan and casein phosphopeptides composite coatings for orthopedic implants. Carbohydrate Polymers. 2015;133:236-44.

[8] Cheng X, Ma K, Li R, Ren X, Huang TS. Antimicrobial coating of modified chitosan onto cotton fabrics. Applied Surface Science. 2014;309:138-43.

[9] Tang S, Tian B, Guo Y-J, Zhu Z-A, Guo Y-P. Chitosan/carbonated hydroxyapatite composite coatings: Fabrication, structure and biocompatibility. Surface and Coatings Technology. 2014;251:210-6.

[10] Yang T-L, Hsiao Y-C. Chitosan facilitates structure formation of the salivary gland by regulating the basement membrane components. Biomaterials. 2015;66:29-40.

[11] Li Z, Dou H, Fu Y, Qin M. Improving the hydrogen peroxide bleaching efficiency of aspen chemithermomechanical pulp by using chitosan. Carbohydrate Polymers. 2015;132:430-6.

[12] Olicón-Hernández DR, Hernández-Lauzardo AN, Pardo JP, Peña A, Velázquez-del Valle MG, Guerra-Sánchez G. Influence of chitosan and its derivatives on cell development and physiology of Ustilago maydis. International Journal of Biological Macromolecules. 2015;79:654-60.

[13] Araújo APC, Venturelli BC, Santos MCB, Gardinal R, Cônsolo NRB, Calomeni GD, et al. Chitosan affects total nutrient digestion and ruminal fermentation in Nellore steers. Animal Feed Science and Technology. 2015;206:114-8.

[14] Rafat M, Li F, Fagerholm P, Lagali NS, Watsky MA, Munger R, et al. PEG-stabilized carbodiimide crosslinked collagen-chitosan hydrogels for corneal tissue engineering. Biomaterials. 2008;29:3960-72. 
[15] Ma X-Y, Feng Y-F, Ma Z-S, Li X, Wang J, Wang L, et al. The promotion of osteointegration under diabetic conditions using chitosan/hydroxyapatite composite coating on porous titanium surfaces. Biomaterials. 2014;35:7259-70.

[16] García MA, Pérez L, de la Paz N, González J, Rapado M, Casariego A. Effect of molecular weight reduction by gamma irradiation on chitosan film properties. Materials Science and Engineering: C. 2015;55:174-80.

[17] Esmaeili A, Beni AA. A novel fixed-bed reactor design incorporating an electrospun PVA/chitosan nanofiber membrane. Journal of Hazardous Materials. 2014;280:788-96.

[18] Mishra SK, Ferreira JMF, Kannan S. Mechanically stable antimicrobial chitosan-PVA-silver nanocomposite coatings deposited on titanium implants. Carbohydrate Polymers. 2015;121:37-48. [19] Paipitak K, Pornpra T, Mongkontalang P, Techitdheer W, Pecharapa W. Characterization of PVA-Chitosan Nanofibers Prepared by Electrospinning. Procedia Engineering. 2011;8:101-5.

[20] Hassani Najafabadi A, Abdouss M, Faghihi S. Synthesis and evaluation of PEG-O-chitosan nanoparticles for delivery of poor water soluble drugs: Ibuprofen. Materials Science and Engineering: C. 2014;41:91-9.

[21] Lin Z, Liu S, Sun X, Xie M, Li J, Li X, et al. The effects of citric acid on the synthesis and performance of silver-tin oxide electrical contact materials. Journal of Alloys and Compounds. 2014;588:30-5.

[22] Sánchez-Ferrero A, Mata Á, Mateos-Timoneda MA, Rodríguez-Cabello JC, Alonso M, Planell J, et al. Development of tailored and self-mineralizing citric acid-crosslinked hydrogels for in situ bone regeneration. Biomaterials. 2015;68:42-53. 
[23] Wang X, Chen J, Yan X, Wang X, Zhang J, Huang J, et al. Heavy metal chemical extraction from industrial and municipal mixed sludge by ultrasound-assisted citric acid. Journal of Industrial and Engineering Chemistry. 2015;27:368-72.

[24] Zhao X, Liu Y, Li W, Long K, Wang L, Liu S, et al. Collagen based film with well epithelial and stromal regeneration as corneal repair materials: Improving mechanical property by crosslinking with citric acid. Materials Science and Engineering: C. 2015;55:201-8.

[25] Xu H, Shen L, Xu L, Yang Y. Low-temperature crosslinking of proteins using non-toxic citric acid in neutral aqueous medium: Mechanism and kinetic study. Industrial Crops and Products. 2015;74:234-40.

[26] Gawish SM, Abo El-Ola SM, Ramadan AM, Abou El-Kheir AA. Citric acid used as a crosslinking agent for the grafting of chitosan onto woolen fabric. Journal of Applied Polymer Science. 2012;123:3345-53.

[27] Yamaguchi I, lizuka S, Osaka A, Monma H, Tanaka J. The effect of citric acid addition on chitosan/hydroxyapatite composites. Colloids and Surfaces A: Physicochemical and Engineering Aspects. 2003;214:111-8.

[28] Bagheri M, Younesi H, Hajati S, Borghei SM. Application of chitosan-citric acid nanoparticles for removal of chromium (VI). International Journal of Biological Macromolecules. 2015;80:431-44.

[29] T. Kokubo HT. How useful is SBF in predicting in vivo bone bioactivity? Biomaterials. 2006;27:2907-2915.

[30] Norhidayu M. Z., Rafaqat H., Mohammed R. A. K. Surface modification of yttria stabilized zirconia via polydopamine inspired coating for hydroxyapatite biomineralization .Applied Surface 
Science. 2014;322: 169-176.

[31] Yan P, Wang J, Wang L, Liu B, Lei Z, Yang S. The in vitro biomineralization and cytocompatibility of polydopamine coated carbon nanotubes. Applied Surface Science. 2011;257:4849-55.

[32] Alvaro J. L., Praveen S., Joao F. M. Chitosan/chondroitin sulfate multilayers as supports for calcium phosphate biomineralization. Materials Letters.2014; 121:62-65.

[33] Hu J-X, Ran J-B, Chen S, Shen X-Y, Tong H. Biomineralization-inspired synthesis of chitosan/hydroxyapatite biocomposites based on a novel bilayer rate-controlling model. Colloids and Surfaces B: Biointerfaces. 2015;136:457-64.

[34] I.R. Serra, R. Fradique, M.C.S. Vallejo, T.R. Correia, S.P. Miguel, I.J. Correia. Production and characterization of chitosan /gelatin / $\beta$-TCP scaffolds for improve bone tissue regeneration. Materials Science and Engineering: C. 2015;55:592-604.

[35] Puvaneswary S, Talebian S, Raghavendran HB, Murali MR, Mehrali M, Afifi AM, et al. Fabrication and in vitro biological activity of $\beta$ TCP-Chitosan-Fucoidan composite for bone tissue engineering. Carbohydrate Polymers. 2015;134:799-807. 
Table 1. Surface elemental compositions of chitosan film before and after modification

\begin{tabular}{cccccc}
\hline Sample & $\mathbf{C}(\boldsymbol{\%})$ & $\mathbf{N}(\boldsymbol{\%})$ & $\mathbf{O}(\boldsymbol{\%})$ & $\mathbf{O} / \mathbf{C}$ & $\mathbf{N} / \mathbf{C}$ \\
\hline CS & 69.34 & 3.21 & 27.45 & 0.40 & 0.05 \\
CS-CA & 65.65 & 1.32 & 33.03 & 0.50 & 0.02 \\
\hline
\end{tabular}


Table 2 Relative intensities of the fitted C1s peak of CS and CS-CA film

\begin{tabular}{cccc}
\hline Sample & Position (eV) & Relative intensity (\%) & Possible element state \\
\hline \multirow{3}{*}{ CS } & 283.47 & 62.22 & $-\mathrm{C}-\mathrm{C}-$ \\
& 284.07 & 32.56 & $-\mathrm{C}-\mathrm{N}-$ \\
& 285.27 & 4.42 & $-\mathrm{C}-\mathrm{O}-$ \\
& 287.00 & 0.80 & $-\mathrm{C}=\mathrm{O}-$ \\
\multirow{4}{*}{ CS-CA } & 283.93 & 48.46 & $-\mathrm{C}-\mathrm{C}-$ \\
& 284.50 & 21.22 & $-\mathrm{C}-\mathrm{N}-$ \\
& 285.62 & 24.55 & $-\mathrm{C}-\mathrm{O}-$ \\
& 287.14 & 5.78 & $-\mathrm{C}=\mathrm{O}-$ \\
\hline
\end{tabular}


$\overbrace{\mathrm{O}}^{\mathrm{OH}}+\mathrm{R}_{\mathrm{O}}^{\mathrm{OH}}-\mathrm{N}=\mathrm{C}=\mathrm{N}-\mathrm{R}_{2}$

CA

EDC<smiles></smiles><smiles>[R1]NC(N[R4])OC(=O)C(O)(C(=O)O)C(=O)O</smiles>

CA-EDC<smiles>[R]NC(N[R])OC(=O)C(O)(C(=O)O)C(=O)O</smiles>

CA-EDC<smiles>O=C1CCC(=O)N1O</smiles><smiles>C[14CH2]C</smiles><smiles>O=C1CCC(=O)N1OC(=O)C(O)(C(=O)O)C(=O)O</smiles>

CA-NHS

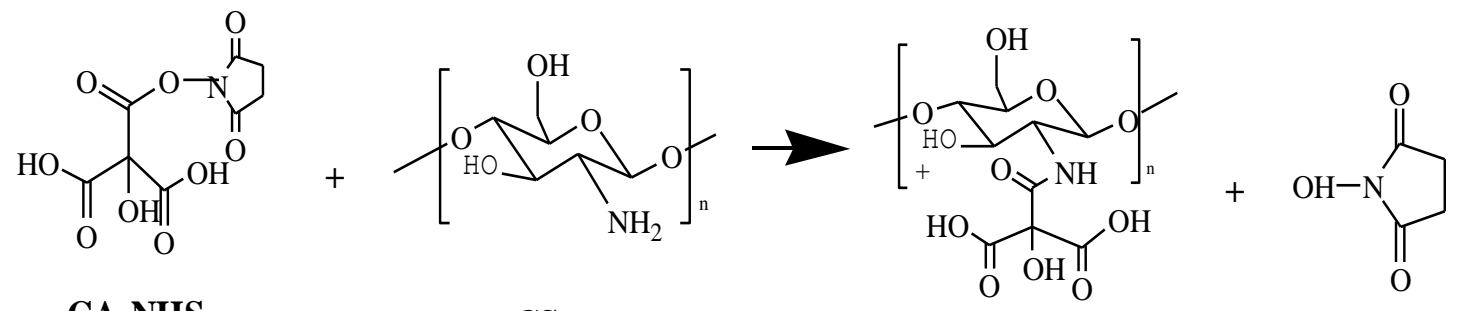

\section{CA-NHS}

CS

CA-CS

NHS

Scheme 1. The schematic illustration of possible reaction routes between chitosan and citric acid,

the cross-linking reaction of chitosan with citric acid is through the condensation polymerization of amine and carboxyl. 


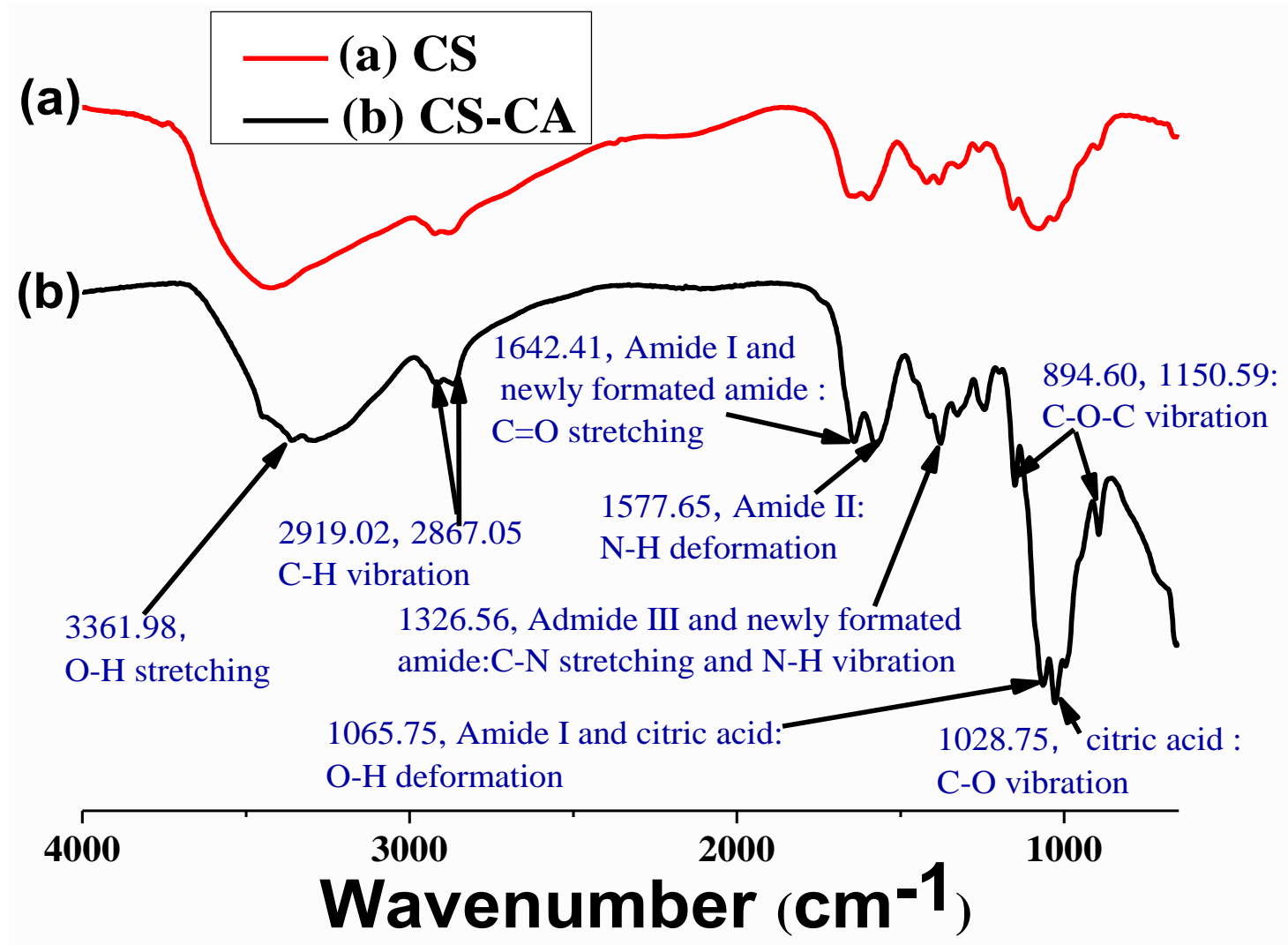

Fig.1. The ATR-FTIR spectrum of the CS (a) and CS-CA film (b). 


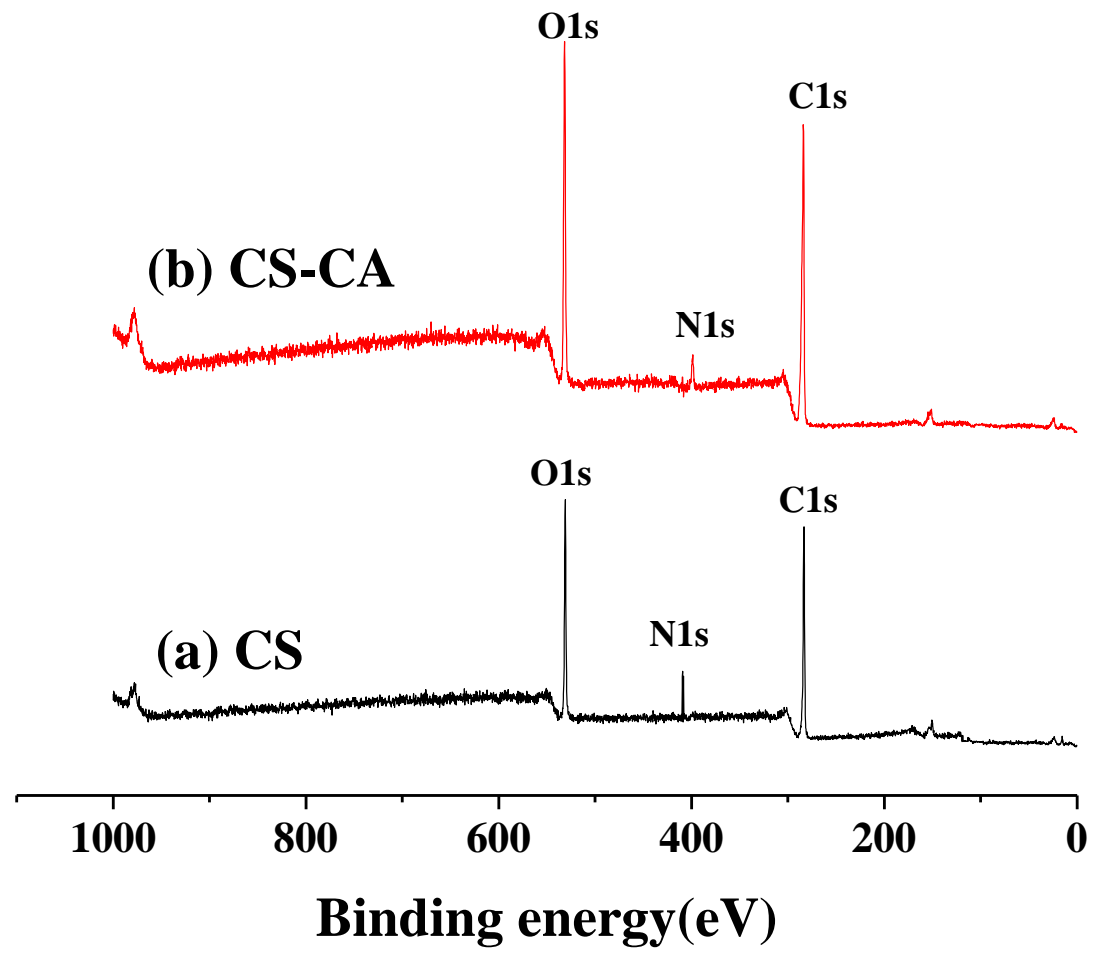

Fig.2. XPS survey spectra of the samples: CS (a) and CS-CA film (b). 

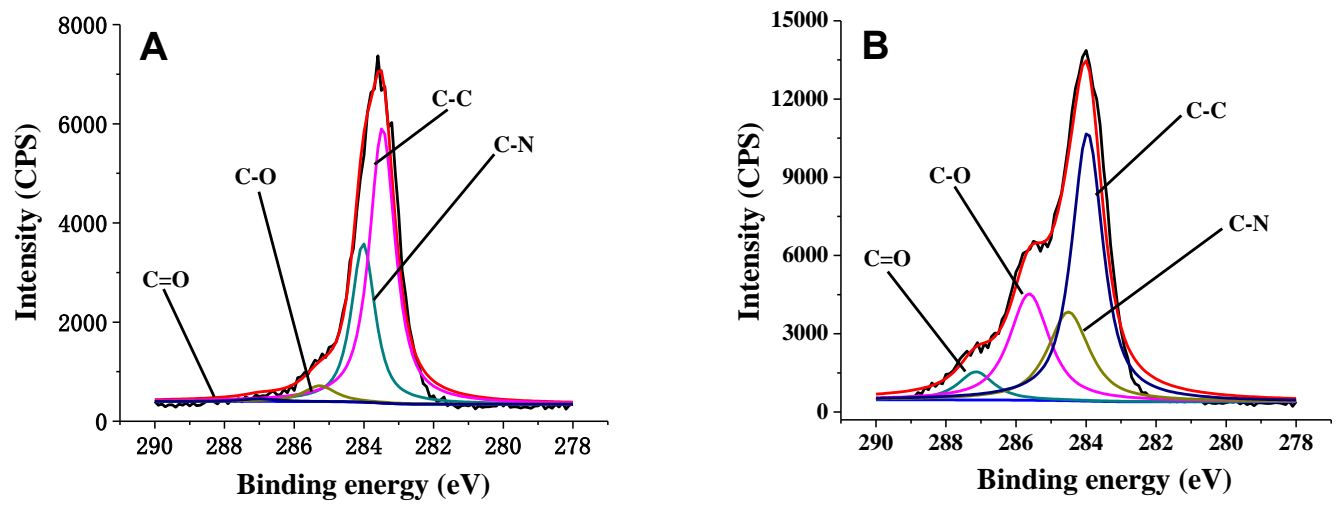

Fig.3. High-resolution XPS spectra of C1s spectra and peak fit analysis for CS (A) and CS-CA film (B). The $\mathrm{C} 1 \mathrm{~s}$ spectrum at $285 \mathrm{eV}$ can be resolved into four main component peaks. 


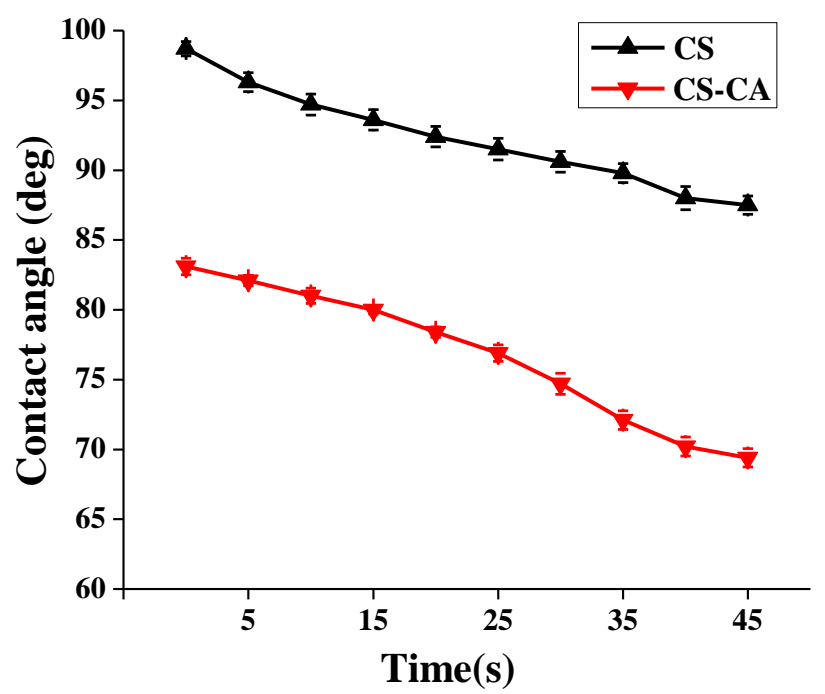

Fig.4. Variation of contact angle versus time for the CS and CS-CA film. Values are expressed as means \pm standard error $(n=5)$. The wettability and hydrophilicity of the film's surface improves with the increase of solid-liquid contact time. 

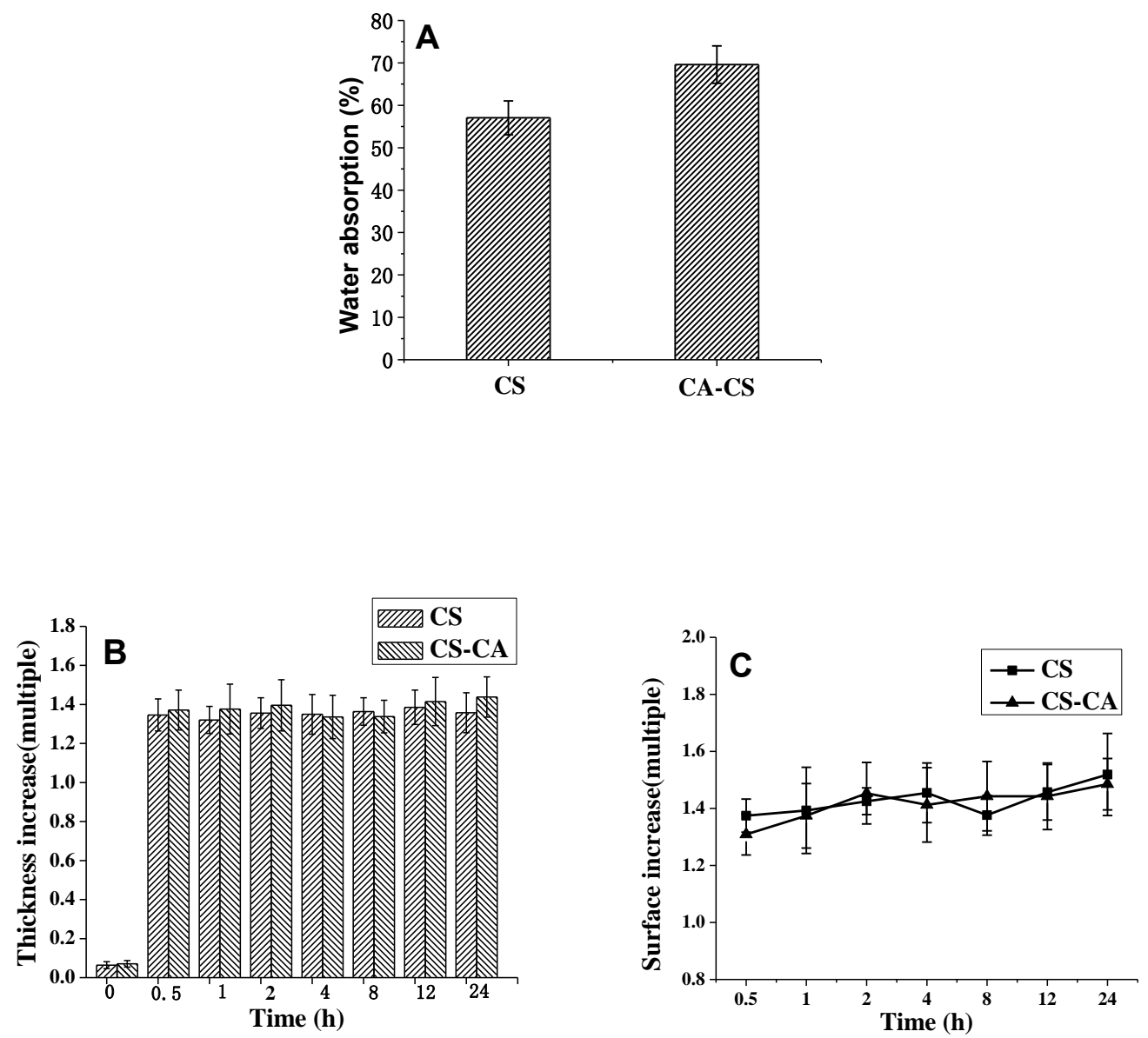

Fig.5. Water absorption percentages of the CS and CS-CA film (A). Variation of the CS and CS-CA film's thickness (B) and surface area (C) versus time. Values are expressed as the means \pm standard error $(\mathrm{n}=10)$. 


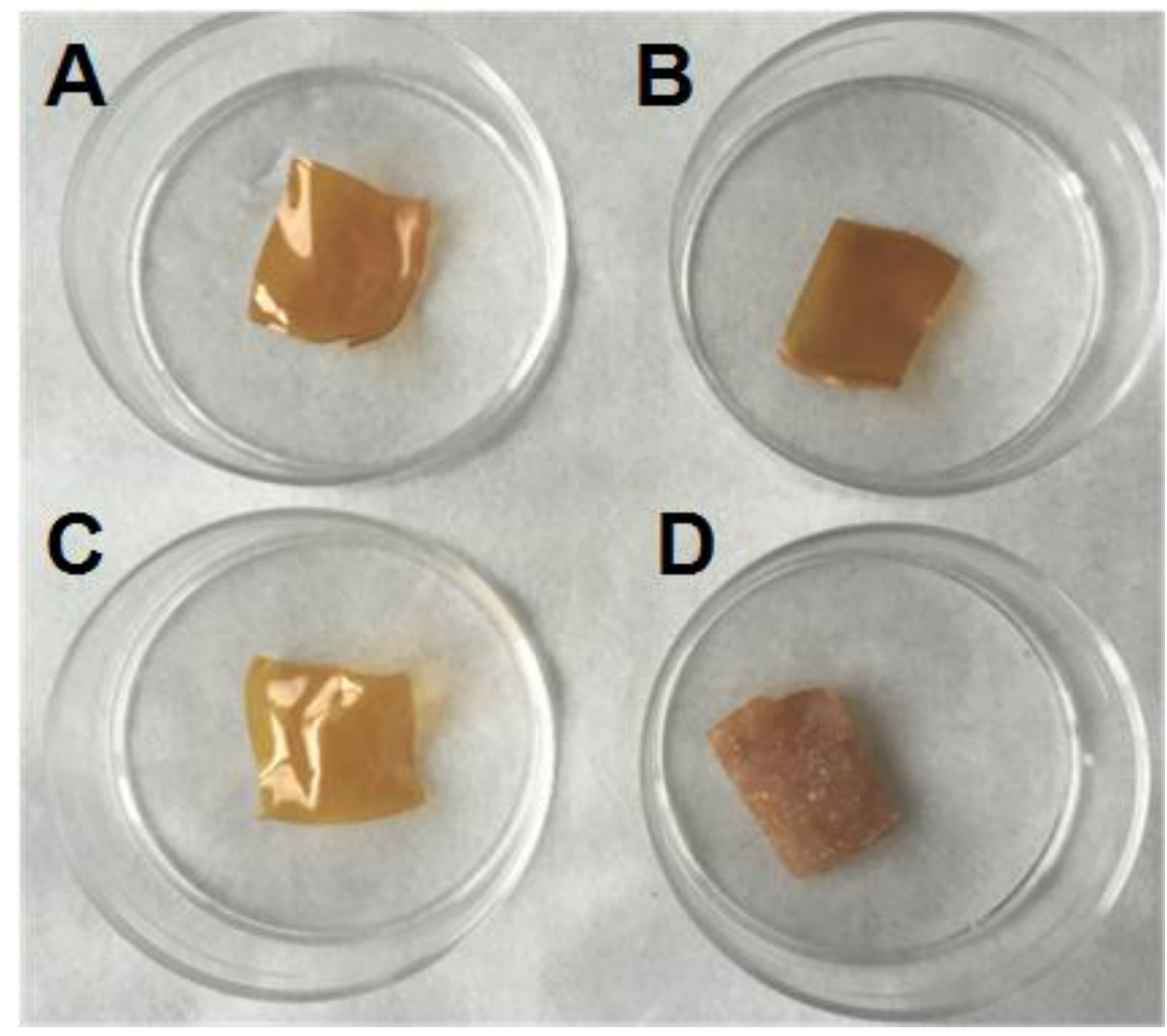

Fig.6. Photograph of the CS film (A, B) and CS-CA film (C, D) after stored in simulated body fluids for 0 (A, C) and 31 days (B, D), a lot of mineralization composition was found on the surface of CS-CA film within 1month. 


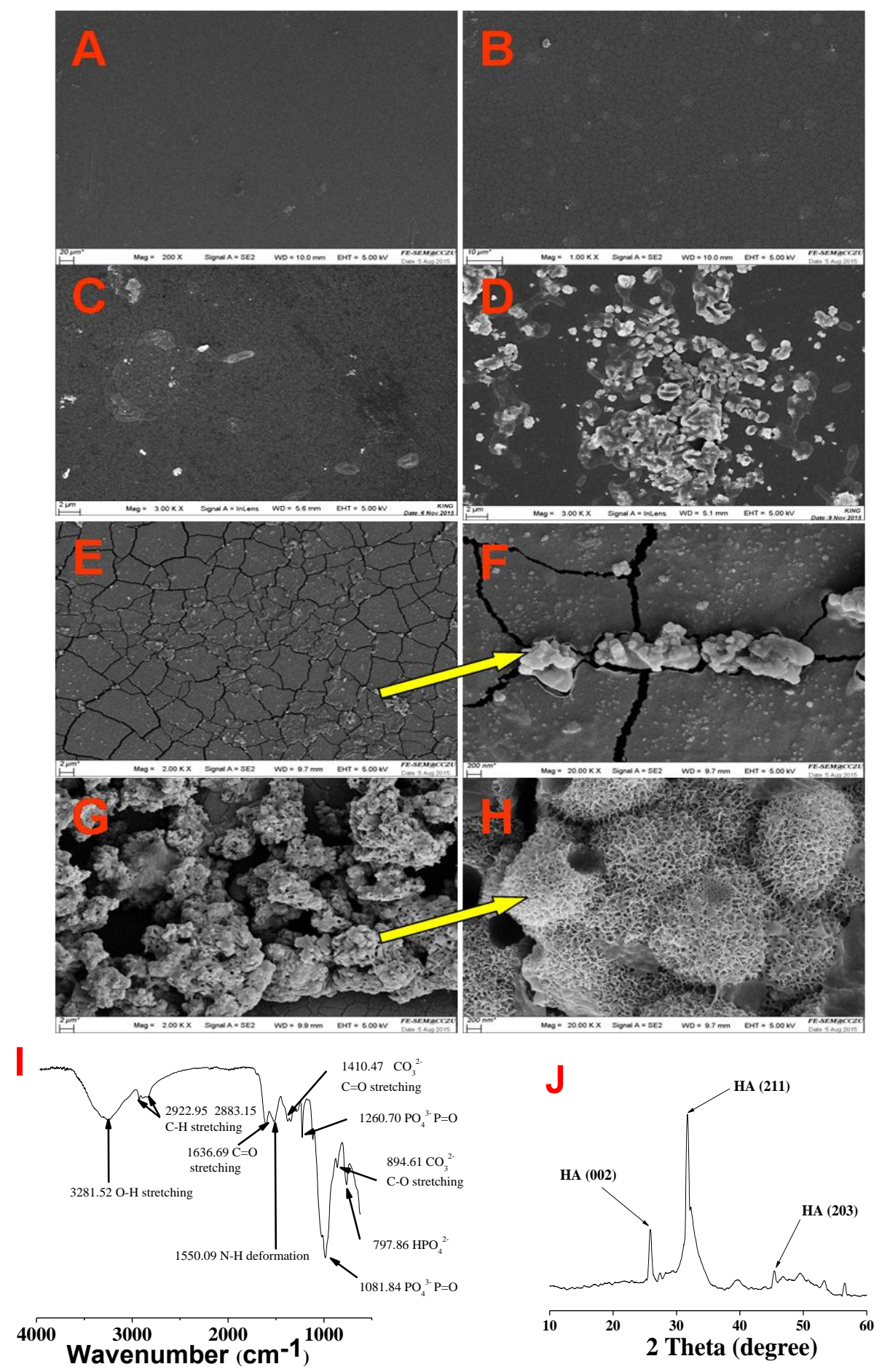

Fig.7. SEM morphology of the CS (A, C, E and F) and CS-CA (B, D, G and H) before and after soaking in SBF. After soaking the samples in SBF, Ca-P salt was formed on the surface of the films at different times (A and B for 0 day, C and D for 2 weeks, E, F, G and $\mathrm{H}$ for 1 month). After being soaked in SBF for 1 month, the FTIR spectra (I) and XRD patterns (J) of the CS-CA film confirmed the formation of crystalline apatite. 

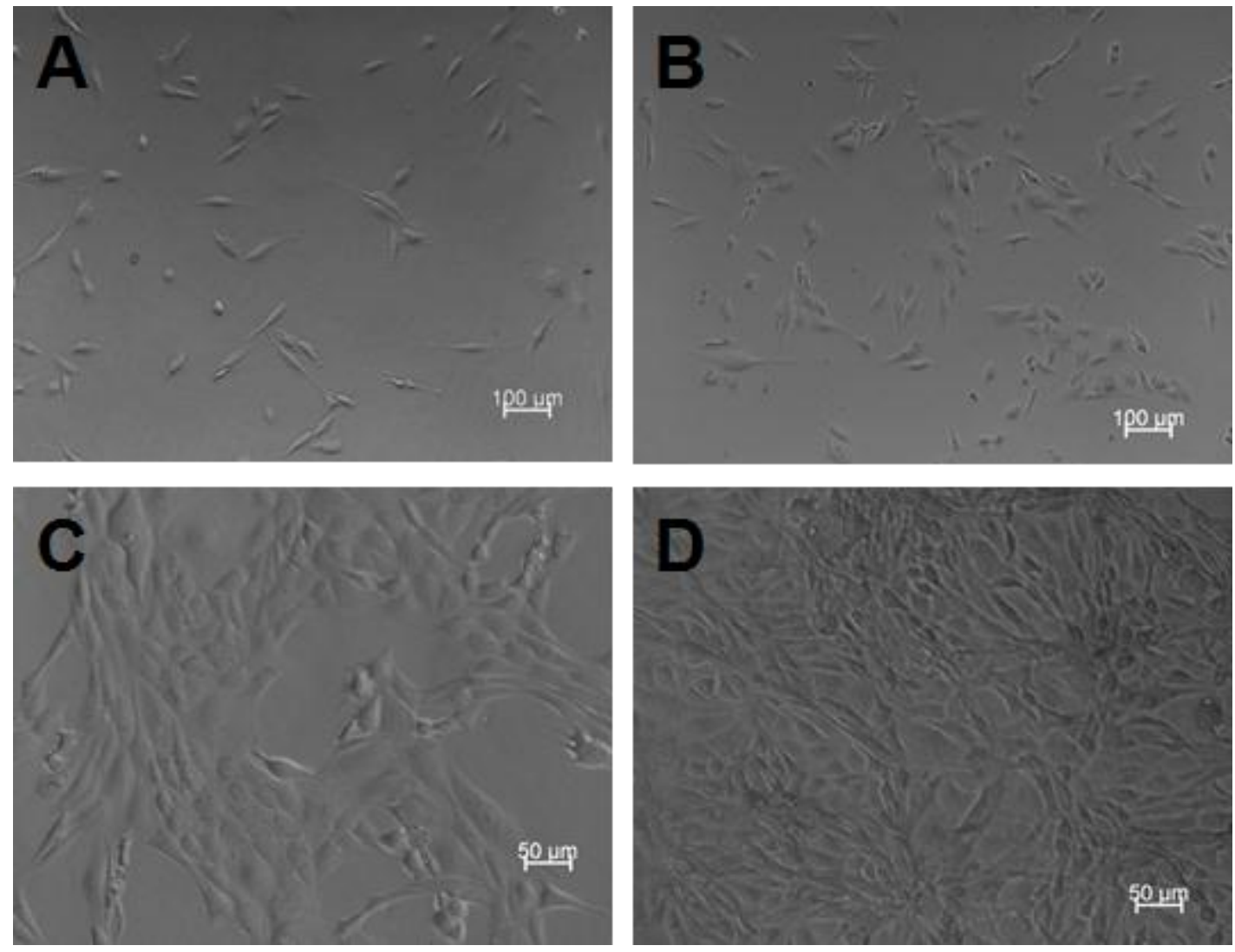

Fig.8. The morphology of MC3T3-E1 on the CS-CA film at different time points, 12h (A), 24h

(B), 48h (C), 72h (D), respectively. The morphology of MC3T3-E1 was changed from round shape to spindle shape gradually. 

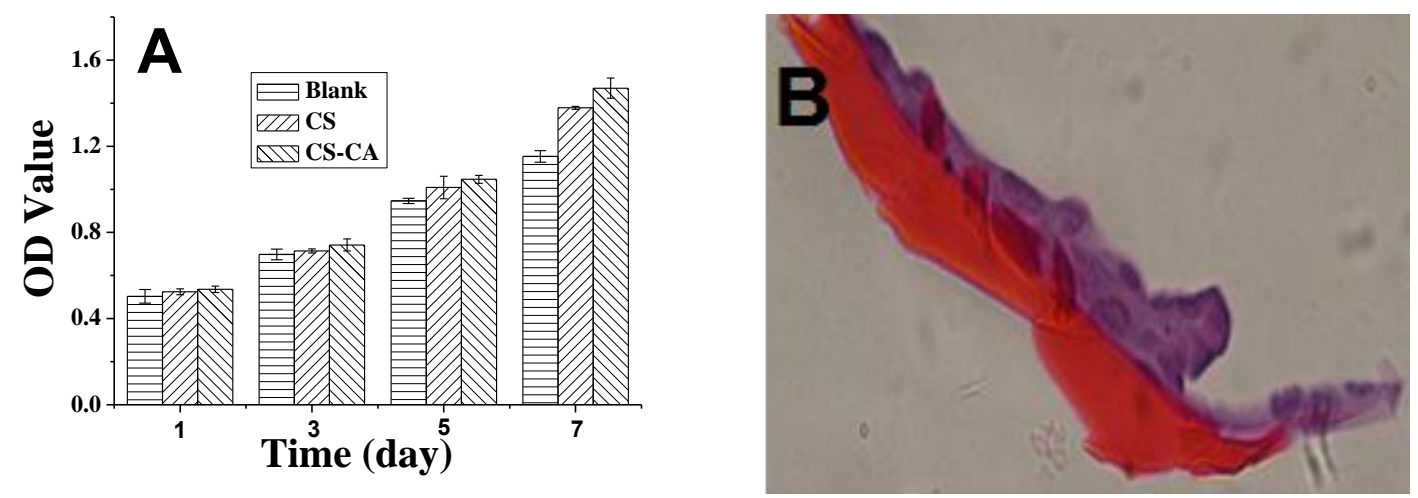

Fig.9. The proliferation of MC3T3-E1 on the CS and CS-CA film (A). Values are expressed as the means \pm standard deviation $(n=10)$. H\&E staining section of MC3T3-E1 constructs grown on CS-CA film consisting of 2-3 layers (B). 


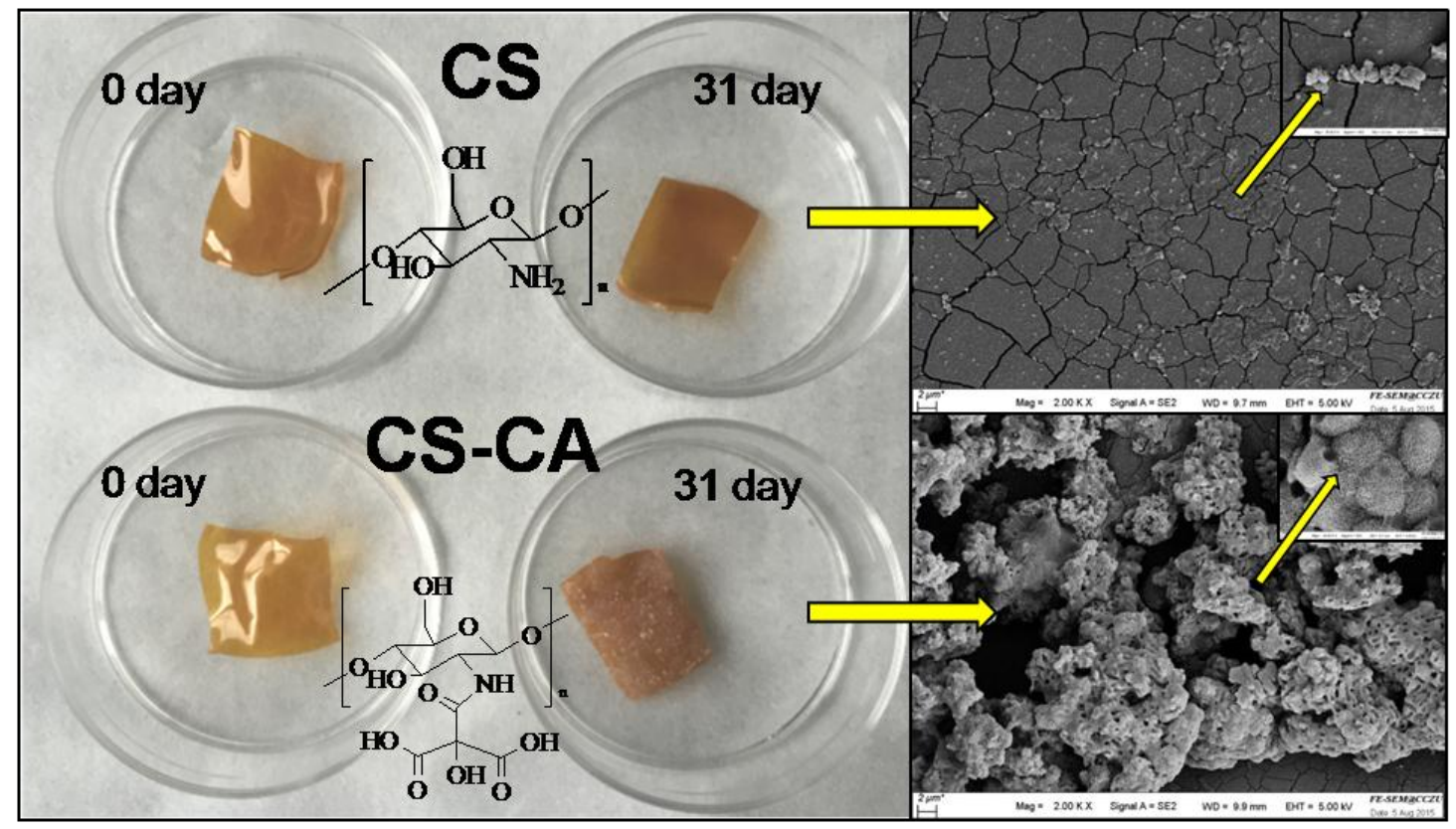

